\title{
TEMPERATURE MODELLING WITHIN A THIN MATERIAL SHEET INVOLVED IN CONDUCTIVE-RADIATIVE HEAT TRANSFER ${ }^{1}$
}

\author{
K. BIRGELIS \\ Institute of Mathematics and Computer Science, University of Latvia \\ 29 Raina Blvd., Riga, Latvia \\ E-mail: karlis_birgelis@navigator.lv
}

Received September 30, 2004; revised May 5, 2005

\begin{abstract}
In this paper we consider a problem about finding of temperature approximation within a thin material sheet involved in conductive-radiative heat transfer. As result, we found that temperature within the sheet can be approximated in $L_{2}$ norm by solution of a simple nonlinear operator equation.
\end{abstract}

Key words: elliptic equation, boundary value problem, thin layer analysis, conductiveradiative heat transfer

\section{Introduction}

Mathematical models for description of conductive-radiative heat transfer are considered in [5]. As a result, complicated integro-differential boundary value problems are derived that models, heat propagation within a physical system.

In [3] a mathematical model is given for the description of process of the oil burn-out from glass fabric sheets. There a glass fabric sheet is pulled with a constant speed through a furnace. As conductive-radiative heat transfer occurs in the heatersfabric sheet system, when the glass fabric is heated up in the furnace. If temperature raises up to the critical level, oil from the fabric starts to burn out. In this paper we neglect oil burn-out process from the fabric and therefore. If we take into account only conductive-radiative heat transfer, then the resulting temperature distribution $T_{\delta}$ in the glass fabric sheet $\Omega_{\delta}$ of thickness $2 \delta$ can be found after solving the following elliptic boundary value problem:

\footnotetext{
${ }^{1}$ This work was supported by Latvian Council of Science under Grant 01.0441.
} 


$$
\begin{aligned}
& k_{1} \Delta T_{\delta}-k_{2} \frac{\partial T_{\delta}}{\partial x_{1}}=0 \text { in } \Omega_{\delta}, \\
& k_{1} \frac{\partial T_{\delta}}{\partial \nu}=-G_{\delta}^{1}\left(\left|T_{\delta}\right|^{3} T_{\delta}\right)+G_{\delta}^{2}\left(\left|T^{h}\right|^{3} T^{h}\right)-k_{3}\left(T_{\delta}-T_{\delta}^{g}\right) \text { on } \Sigma_{\delta}^{s} \subset \partial \Omega_{\delta}, \\
& T_{\delta}=T_{\delta}^{i} \text { on } \Sigma_{\delta}^{i} \subset \Omega_{\delta} \backslash \Sigma_{\delta}^{s}, \quad k_{1} \frac{\partial T_{\delta}}{\partial \nu}=0 \text { on } \partial \Omega_{\delta} \backslash\left(\Sigma_{\delta}^{s} \cup \Sigma_{\delta}^{i}\right) .
\end{aligned}
$$

Here linear operators

$$
G_{\delta}^{1}: L_{5 / 4}\left(\Sigma_{\delta}^{s}\right) \mapsto L_{5 / 4}\left(\Sigma_{\delta}^{s}\right), \quad G_{\delta}^{2}: L_{5 / 4}\left(\Sigma^{h}\right) \mapsto L_{5 / 4}\left(\Sigma_{\delta}^{s}\right)
$$

are given in the implicit form and they describe radiative heat propagation within $\Sigma_{\delta}^{s}$, $\Sigma^{h}$ system, where $\Sigma_{\delta}^{s}$ is active surface of $\Omega_{\delta}$ and $\Sigma^{h}$ is active surface of the furnace. Both surfaces can emit, absorb and reflect radiation. $T^{h}$ is temperature distribution on $\Sigma^{h}$, whereas $T_{\delta}^{g}$ is temperature of the surrounding air.

Unfortunately, it is very hard to carry out any numerical calculations for the boundary value problem (1.1) - (1.3) with standard numerical methods. The main reason is the fact that the sheet geometry is strongly degenerated in this situation. Thickness-width ratio for the sheet can achieve the value $\frac{1}{15000}$.

In this paper we have analyzed the dependence of the weak solutions $T_{\delta}$ of the boundary value problem (1.1) - (1.3) on the thickness $2 \delta$ and found, that, as $\delta \rightarrow 0$, the weak solutions $T_{\delta}$ can be approximated in $L_{2}\left(\Omega_{\delta}\right)$ norm by solutions of a simple nonlinear operator equation.

\section{Preliminaries}

In this section we give the formulation of the full integro-differential boundary value problem and list some preliminary results. It is important to note that we widely use the methodology from paper [5], which deals with similar mathematical models of conductive-radiative heat transfer. Let

$$
\Omega_{\delta}:=\left(-l_{1}, l_{1}\right) \times\left(-l_{2}, l_{2}\right) \times(-\delta, \delta)
$$

be a glass fabric sheet of thickness $2 \delta\left(l_{1}>0, l_{2}>0, l_{3}>0,0<\delta \leq l_{3}\right)$ pulled in $x_{1}$ axis direction through a furnace. Let us denote faces of $\Omega_{\delta}$ by:

$$
\begin{aligned}
& \Sigma_{\delta}^{+}:=\left\{x \in \partial \Omega_{\delta}: x_{3}=\delta\right\}, \quad \Sigma_{\delta}^{-}:=\left\{x \in \partial \Omega_{\delta}: x_{3}=-\delta\right\}, \\
& \Sigma_{\delta}^{\prime}:=\left\{x \in \partial \Omega_{\delta}: x_{2}=-l_{2}\right\}, \quad \Sigma_{\delta}^{\prime \prime}:=\left\{x \in \partial \Omega_{\delta}: x_{2}=l_{2}\right\}, \\
& \Sigma_{\delta}^{i}:=\left\{x \in \partial \Omega_{\delta}: x_{1}=-l_{1}\right\}, \quad \Sigma_{\delta}^{o}:=\left\{x \in \partial \Omega_{\delta}: x_{1}=l_{1}\right\} .
\end{aligned}
$$

We assume that only one part of $\partial \Omega_{\delta}$ is involved in radiative heat exchange:

$$
\Sigma_{\delta}^{s}:=\Sigma_{\delta}^{-} \cup \Sigma_{\delta}^{+} \cup \Sigma_{\delta}^{\prime} \cup \Sigma_{\delta}^{\prime \prime} .
$$

Let the furnace is formed from finite set of heaters $\left\{\Omega_{i} \subset \mathbb{R}^{3}: i=1, \ldots, n\right\}$. For mathematical reasons we assume that each heater is a bounded Lipschitz domain with piecewise Lyapunov boundary and it lies at a positive distance from other heaters and from $\Omega_{\delta}$. Let us denote the overall heater surface of the furnace by 


$$
\Sigma^{h}:=\bigcup_{i \in\{1, . ., n\}} \partial \Omega_{i}
$$

Let us also denote

$$
Q:=\left[-l_{1}, l_{1}\right] \times\left[-l_{2}, l_{2}\right], \quad \Sigma_{\delta}^{r}:=\Sigma_{\delta}^{s} \cup \Sigma^{h}, \quad \Omega_{\delta}^{r}:=\left(\bigcup_{i \in\{1, . ., n\}} \Omega_{i}\right) \cup \Omega_{\delta} .
$$

In what follows $c$ and $M$ with subscripts will denote nonnegative constants that do not depend on $\delta$.

We denote by $\mathfrak{L}(X, Y)$ a space of the linear bounded operators that maps a Banach space $X$ into a Banach space $Y$. Let $I$ be an identity operator.

We denote by $L_{p}(\cdot)$ ithe standard Lebesgue spaces $(1 \leq p \leq \infty)$ and by $W_{2}^{1}(\cdot)$, $W_{\infty}^{1}(\cdot)$ the standard Sobolev spaces. Let $V_{5}\left(\Omega_{\delta}\right), \dot{V}_{5}\left(\Omega_{\delta}\right)$ be Banach spaces:

$$
\begin{aligned}
& V_{5}\left(\Omega_{\delta}\right):=\left\{u \in W_{2}^{1}\left(\Omega_{\delta}\right):\left.u\right|_{\left.\Sigma_{\delta}^{s} \in L_{5}\left(\Sigma_{\delta}^{s}\right)\right\},}\right. \\
& \dot{V}_{5}\left(\Omega_{\delta}\right):=\left\{u \in V_{5}\left(\Omega_{\delta}\right):\left.u\right|_{\Sigma_{\delta}^{i}}=0\right\} .
\end{aligned}
$$

If we make substitution $T_{\delta} \mapsto u_{\delta}+\phi_{\delta}$, then we can rewrite the boundary value problem (1.1) - (1.3) in the weak form:

$$
\begin{aligned}
\int_{\Omega_{\delta}}\left(k_{1}\left(\nabla\left(u_{\delta}+\phi_{\delta}\right) \cdot \nabla \psi\right)+k_{2}\left(u_{\delta}+\phi_{\delta}\right)_{x_{1}} \psi\right) d v & \\
& +\int_{\Sigma_{\delta}^{s}} G_{\delta}^{1}\left(\left|u_{\delta}+\phi_{\delta}\right|^{3}\left(u_{\delta}+\phi_{\delta}\right)\right) \psi d s+\int_{\Sigma_{\delta}^{s}} k_{3}\left(u_{\delta}+\phi_{\delta}\right) \psi d s \\
& =\int_{\Sigma_{\delta}^{s}} G_{\delta}^{2}\left(\left|T^{h}\right|^{3} T^{h}\right) \psi d s+\int_{\Sigma_{\delta}^{s}} k_{3} T_{\delta}^{g} \psi d s, \quad \forall \psi \in \dot{V}_{5}\left(\Omega_{\delta}\right),
\end{aligned}
$$

where $u_{\delta} \in \dot{V}_{5}\left(\Omega_{\delta}\right)$ is unknown variable and

$$
\begin{aligned}
& k_{1}>0, \quad k_{2}>0, \quad k_{3}>0, \quad \phi_{\delta} \in V_{5}\left(\Omega_{\delta}\right), \phi_{\delta}=T_{\delta}^{i} \text { a.e. on } \Sigma_{\delta}^{i}, \\
& T_{\delta}^{i} \in W_{\infty}^{1}\left(\Sigma_{\delta}^{i}\right), \quad 0 \leq T_{\delta}^{i} \leq M_{1} \text { a.e. on } \Sigma_{\delta}^{i}, \\
& T^{h} \in L_{\infty}\left(\Sigma^{h}\right), \quad 0 \leq T^{h} \leq M_{1} \text { a.e. on } \Sigma^{h}, \\
& T_{\delta}^{g} \in L_{\infty}\left(\Sigma_{\delta}^{s}\right), \quad 0 \leq T_{\delta}^{g} \leq M_{1} \text { a.e. on } \Sigma_{\delta}^{s}, \\
& G_{\delta}^{1} \in \mathfrak{L}\left(L_{5 / 4}\left(\Sigma_{\delta}^{s}\right), L_{5 / 4}\left(\Sigma_{\delta}^{s}\right)\right), \quad G_{\delta}^{2} \in \mathfrak{L}\left(L_{5 / 4}\left(\Sigma^{h}\right), L_{5 / 4}\left(\Sigma_{\delta}^{s}\right)\right) .
\end{aligned}
$$

Further we assume that $\left.T_{\delta}^{g}\right|_{\Sigma_{\delta}^{+}},\left.T_{\delta}^{g}\right|_{\Sigma_{\delta}^{-}}$do not depend on $\delta$. We also assume that $\left.T_{\delta}^{g}\right|_{\Sigma_{\delta}^{\prime}},\left.T_{\delta}^{g}\right|_{\Sigma_{\delta}^{\prime \prime}}$ depend only on variable $x_{1}, T_{\delta}^{i}$ depend only on variable $x_{2}$ and there exists a constant $M_{2}$ such that

$$
\left(\left.T_{\delta}^{g}\right|_{\Sigma}\right) \in W_{\infty}^{1}(\Sigma), \quad\left\|\left(\left.T_{\delta}^{g}\right|_{\Sigma}\right)\right\|_{W_{\infty}^{1}(\Sigma)} \leq M_{2},
$$

if $\Sigma$ is one of the surfaces $\Sigma_{\delta}^{+}, \Sigma_{\delta}^{-}, \Sigma_{\delta}^{\prime}$ and $\Sigma_{\delta}^{\prime \prime}$. 
Originally mathematical formalization of conductive-radiative heat exchange process in the furnace-fabric sheet system gives us the following system:

$$
\begin{gathered}
\int_{\Omega_{\delta}}\left(k_{1}\left(\nabla\left(u_{\delta}+\phi_{\delta}\right) \cdot \nabla \psi\right)+k_{2}\left(u_{\delta}+\phi_{\delta}\right)_{x_{1}} \psi\right) d v+\int_{\Sigma_{\delta}^{s}} k_{3}\left(u_{\delta}+\phi_{\delta}\right) \psi d s \\
\quad+\int_{\Sigma_{\delta}^{s}} g_{\delta} \psi d s=\int_{\Sigma_{\delta}^{s}} k_{3} T_{\delta}^{g} \psi d s \quad \forall \psi \in \dot{V}_{5}\left(\Omega_{\delta}\right) \\
\rho_{\delta}(x)-\left(1-\epsilon_{\delta}(x)\right) \int_{\Sigma_{\delta}^{r}} k_{\delta}(x, y) \rho_{\delta}(y) d s(y) \\
g_{\delta}(x)=\rho_{\delta}(x)-\int_{\Sigma_{\delta}^{r}} k_{\delta}(x, y) \rho_{\delta}(y) d s(y) \text { a.e. }\left.x \in q_{\delta}(x)\right|^{3} q_{\delta}(x) \text { a.e. } x \in \Sigma_{\delta}^{r}, \\
q_{\delta}\left|\Sigma_{\delta}^{s}=u_{\delta}+\phi_{\delta}, \quad q_{\delta}\right| \Sigma^{h}=T^{h},
\end{gathered}
$$

where

$$
\sigma>0, \epsilon_{0}>0, \epsilon_{\delta} \in L_{\infty}\left(\Sigma_{\delta}^{r}\right), \epsilon_{0} \leq \epsilon_{\delta} \leq 1 \text { a.e. on } \Sigma_{\delta}^{r} .
$$

However, under some assumptions this system can be transformed into the boundary value problem (2.1) ((1.1) - (1.3)).

Further we assume that the functions $\left.\epsilon_{\delta}\right|_{\Sigma_{\delta}^{+}},\left.\epsilon_{\delta}\right|_{\Sigma_{\delta}^{-}}$do not depend on $\delta$ and let us denote , them as $\epsilon^{+}$and $\epsilon^{-}$respectively. We also assume that $\left.\epsilon_{\delta}\right|_{\Sigma_{\delta}^{\prime}},\left.\epsilon_{\delta}\right|_{\Sigma_{\delta}^{\prime \prime}}$ depend only on variable $x_{1}$ and there exits a constant $M_{3}$ such that

$$
\left(\left.\epsilon_{\delta}\right|_{\Sigma}\right) \in W_{\infty}^{1}(\Sigma), \quad\left\|\left(\left.\epsilon_{\delta}\right|_{\Sigma}\right)\right\|_{W_{\infty}^{1}(\Sigma)} \leq M_{3},
$$

if $\Sigma$ is one of the surfaces $\Sigma_{\delta}^{+}, \Sigma_{\delta}^{-}, \Sigma_{\delta}^{\prime}$ and $\Sigma_{\delta}^{\prime \prime}$.

The kernel $k_{\delta}: \Sigma_{\delta}^{r} \times \Sigma_{\delta}^{r} \mapsto \mathbb{R}$ from (2.3), (2.4) is defined as

$$
\begin{aligned}
& k_{\delta}(x, y):=w_{\delta}(x, y) \theta_{\delta}(x, y), \\
& w_{\delta}(x, y):=\frac{\cos (\nu(x),(y-x)) \cos (\nu(y),(x-y))}{\pi|x-y|^{2}}, \\
& \theta_{\delta}(x, y):= \begin{cases}1, & \text { if }\left\{z \in \mathbb{R}^{3}: z=\tau x+(1-\tau) y, 0<\tau<1\right\} \cap \Omega_{\delta}^{r}=\emptyset, \\
0, & \text { otherwise, }\end{cases}
\end{aligned}
$$

where $\nu(\cdot)$ denotes the outward normal of the surface $\Sigma_{\delta}^{r}(\nu(\cdot)$ exists almost everywhere on $\Sigma_{\delta}^{r}$, as it is Lipschitz surface).

The analytical properties of the function $k_{\delta}(x, y)$ and the fact that $\Sigma_{\delta}^{r}$ is Lipschitz surface allow us by involving the Gauss's formula to get the following estimate:

$$
\begin{aligned}
& 0 \leq \int_{\Sigma_{\delta}^{r}} k_{\delta}(x, y) d s(y)=\lim _{\tau \rightarrow 0} \int_{\Sigma_{\delta}^{r} \backslash B(x, \tau)} k_{\delta}(x, y) d s(y) \\
& \quad=\lim _{\tau \rightarrow 0} \int_{S^{+}(x, \tau) \cap Q(x, \tau)} \frac{\cos (\nu(x),(y-x))}{\pi \tau^{2}} d s(y) \leq 1 \text { a.e. } x \in \Sigma_{\delta}^{r},
\end{aligned}
$$


where

$$
\begin{aligned}
& B(x, \tau):=\left\{z \in \mathbb{R}^{3}:|z-x| \leq \tau\right\} \\
& S^{+}(x, \tau):=\left\{z \in \mathbb{R}^{3}:|z-x|=\tau,(\nu(x) \cdot(z-x)) \geq 0\right\} \\
& Q(x, \tau):=\left\{z \in \mathbb{R}^{3}: \quad z=x+t y, y \in \Sigma_{\delta}^{r} \backslash B(x, \tau), t \geq 0\right\}
\end{aligned}
$$

Cylindrical shape of the domain $\Omega_{\delta}$ and positive distance between the surfaces $\Sigma_{\delta}^{s}$, $\Sigma^{h}$ guaranty that there exists a constant $0 \leq c_{1}<1$ such that for every $\tau>0$ and for a.e. $x \in \Sigma_{\delta}^{s}$ the following estimate holds:

$$
\operatorname{meas}\left(S^{+}(x, \tau) \cap Q(x, \tau)\right) \leq 2 c_{1} \tau^{2} .
$$

That yields the existence of a much stronger local estimate on the surface $\Sigma_{\delta}^{s}$ than (2.6). Respectively, there exists a constant $c_{2}<1$ such that:

$$
0 \leq \int_{\Sigma_{\delta}^{r}} k_{\delta}(x, y) d s(y) \leq c_{2} \text { a.e. } x \in \Sigma_{\delta}^{s} .
$$

The estimate (2.6) implies that the operator $K_{\delta}(u):=\int_{\Sigma_{\delta}^{r}} k_{\delta}(x, y) u(y) d s(y)$ maps $L_{p}\left(\Sigma_{\delta}^{r}\right)$ into $L_{p}\left(\Sigma_{\delta}^{r}\right), K_{\delta} \in \mathfrak{L}\left(L_{p}\left(\Sigma_{\delta}^{r}\right), L_{p}\left(\Sigma_{\delta}^{r}\right)\right)$ and

$$
\left\|K_{\delta}\right\|_{\mathfrak{L}\left(L_{p}\left(\Sigma_{\delta}^{r}\right), L_{p}\left(\Sigma_{\delta}^{r}\right)\right)} \leq 1
$$

for every $1 \leq p \leq \infty$ ([4]). For a fixed measurable set $\Sigma \subset \Sigma_{\delta}^{r}$ we define operators

$$
\begin{aligned}
& P_{\delta}^{1}(\Sigma) \in \mathfrak{L}\left(L_{5 / 4}(\Sigma), L_{5 / 4}\left(\Sigma_{\delta}^{r}\right)\right), \quad P_{\delta}^{2}(\Sigma) \in \mathfrak{L}\left(L_{5 / 4}\left(\Sigma_{\delta}^{r}\right), L_{5 / 4}(\Sigma)\right), \\
& E_{\delta}^{1} \in \mathfrak{L}\left(L_{5 / 4}\left(\Sigma_{\delta}^{r}\right), L_{5 / 4}\left(\Sigma_{\delta}^{r}\right)\right), \quad E_{\delta}^{2}(\Sigma) \in \mathfrak{L}\left(L_{5 / 4}(\Sigma), L_{5 / 4}\left(\Sigma_{\delta}^{r}\right)\right), \\
& F_{\delta} \in \mathfrak{L}\left(L_{5 / 4}\left(\Sigma_{\delta}^{r}\right), L_{5 / 4}\left(\Sigma_{\delta}^{r}\right)\right),
\end{aligned}
$$

where

$$
\begin{aligned}
& P_{\delta}^{1}(\Sigma)(u):=\left\{\begin{array}{c}
u(x) \quad x \in \Sigma, \\
0 \quad x \in \Sigma_{\delta}^{r} \backslash \Sigma,
\end{array} \quad P_{\delta}^{2}(\Sigma)(u):=\left.u\right|_{\Sigma},\right. \\
& E_{\delta}^{1}(u):=\left(1-\epsilon_{\delta}\right) u, \quad E_{\delta}^{2}(\Sigma)(u):=P_{\delta}^{1}(\Sigma)\left(\left.\sigma \epsilon_{\delta}\right|_{\Sigma} u\right), \\
& F_{\delta}(u):=E_{\delta}^{2}\left(\Sigma_{\delta}^{r}\right) K_{\delta}\left(\sum_{i=0}^{\infty}\left(E_{\delta}^{1} K_{\delta}\right)^{i}\right)(u) .
\end{aligned}
$$

As estimate (2.8) holds, then it allows us to exclude the variables $g_{\delta}, \rho_{\delta}, q_{\delta}$ from system (2.2)-(2.5) and to obtain the integral equality (2.1). Then operators $G_{\delta}^{1}, G_{\delta}^{2}$ from (2.1) will have the following form ([5]):

$$
G_{\delta}^{1}=P_{\delta}^{2}\left(\Sigma_{\delta}^{s}\right)\left(I-F_{\delta}\right) E_{\delta}^{2}\left(\Sigma_{\delta}^{s}\right), \quad G_{\delta}^{2}=P_{\delta}^{2}\left(\Sigma_{\delta}^{s}\right) F_{\delta} E_{\delta}^{2}\left(\Sigma^{h}\right)
$$

and for $\Sigma_{1}, \Sigma_{2} \subset \Sigma_{\delta}^{s}$, then the following will hold ([5]):

$$
P_{\delta}^{2}\left(\Sigma_{2}\right) F_{\delta} E_{\delta}^{2}\left(\Sigma_{1}\right) \in \mathfrak{L}\left(L_{p}\left(\Sigma_{1}\right), L_{p}\left(\Sigma_{2}\right)\right) \text { for all } 5 / 4 \leq p \leq \infty,
$$


$P_{\delta}^{2}\left(\Sigma_{2}\right) F_{\delta} E_{\delta}^{2}\left(\Sigma^{h}\right) \in \mathfrak{L}\left(L_{p}\left(\Sigma^{h}\right), L_{p}\left(\Sigma_{2}\right)\right)$ for all $5 / 4 \leq p \leq \infty$, $P_{\delta}^{2}\left(\Sigma_{2}\right) F_{\delta} P_{\delta}^{1}\left(\Sigma_{1}\right) \in \mathfrak{L}\left(L_{p}\left(\Sigma_{1}\right), L_{p}\left(\Sigma_{2}\right)\right)$ for all $5 / 4 \leq p \leq \infty$, $\left\|P_{\delta}^{2}\left(\Sigma_{2}\right) F_{\delta} P_{\delta}^{2}\left(\Sigma_{1}\right)\right\|_{\mathfrak{L}\left(L_{p}\left(\Sigma_{1}\right), L_{p}\left(\Sigma_{2}\right)\right)} \leq c_{3}<1$ for all $5 / 4 \leq p \leq \infty$,

if $u \in L_{5 / 4}\left(\Sigma^{h}\right)$ and $u \geq 0$ a.e. on $\Sigma^{h}$, then $P_{\delta}^{2}\left(\Sigma_{2}\right) F_{\delta} E_{\delta}^{2}\left(\Sigma^{h}\right)(u) \geq 0$ a.e. on $\Sigma_{2}$, if $u \in L_{5 / 4}\left(\Sigma_{1}\right)$ and $u \geq 0$ a.e. on $\Sigma_{1}$, then $P_{\delta}^{2}\left(\Sigma_{2}\right) F_{\delta} E_{\delta}^{2}\left(\Sigma_{1}\right)(u) \geq 0$ a.e. on $\Sigma_{2}$.

Moreover, one can show that, if $u_{1} \in L_{\infty}(\Sigma),\left\|u_{1}\right\|_{L_{\infty}(\Sigma)} \leq M_{1}, u_{2} \in$ $L_{\infty}\left(\Sigma_{\delta}^{s}\right),\left\|u_{2}\right\|_{L_{\infty}\left(\Sigma_{\delta}^{s}\right)} \leq M_{1}, u_{3} \in L_{\infty}\left(\Sigma^{h}\right),\left\|u_{3}\right\|_{L_{\infty}\left(\Sigma^{h}\right)} \leq M_{1}$ and $\Sigma$ is one of the $\Sigma_{\delta}^{+}, \Sigma_{\delta}^{-}, \Sigma_{\delta}^{\prime}, \Sigma_{\delta}^{\prime \prime}$, then there exists a constant $M_{4} \geq 0$, such that

$$
\begin{aligned}
& P_{\delta}^{2}(\Sigma) F_{\delta} E_{\delta}^{2}(\Sigma)\left(u_{1}\right) \in W_{\infty}^{1}(\Sigma),\left\|P_{\delta}^{2}(\Sigma) F_{\delta} E_{\delta}^{2}(\Sigma)\left(u_{1}\right)\right\|_{W_{\infty}^{1}(\Sigma)} \leq M_{4}, \\
& P_{\delta}^{2}(\Sigma) F_{\delta} E_{\delta}^{2}\left(\Sigma_{\delta}^{s}\right)\left(u_{2}\right) \in W_{\infty}^{1}(\Sigma),\left\|P_{\delta}^{2}(\Sigma) F_{\delta} E_{\delta}^{2}\left(\Sigma_{\delta}^{s}\right)\left(u_{2}\right)\right\|_{W_{\infty}^{1}(\Sigma)} \leq M_{4}, \\
& P_{\delta}^{2}(\Sigma) F_{\delta} E_{\delta}^{2}\left(\Sigma^{h}\right)\left(u_{3}\right) \in W_{\infty}^{1}(\Sigma),\left\|P_{\delta}^{2}(\Sigma) F_{\delta} E_{\delta}^{2}\left(\Sigma^{h}\right)\left(u_{3}\right)\right\|_{W_{\infty}^{1}(\Sigma)} \leq M_{4} .
\end{aligned}
$$

If we adapt results from [1], [2], then we can get existence and uniform boundedness of solutions for the boundary value problem (2.1). As the form

$$
\begin{aligned}
& \left(\left(u_{\delta}, \psi\right) \in \dot{V}_{5}\left(\Omega_{\delta}\right) \times \dot{V}_{5}\left(\Omega_{\delta}\right)\right) \mapsto \\
& \quad \int_{\Omega_{\delta}}\left(k_{1}\left(\nabla\left(u_{\delta}+\phi_{\delta}\right) \cdot \nabla \psi\right)+k_{2}\left(u_{\delta}+\phi_{\delta}\right)_{x_{1}} \psi\right) d v+\int_{\Sigma_{\delta}^{s}} k_{3}\left(u_{\delta}+\phi_{\delta}\right) \psi d s
\end{aligned}
$$

defines a monotone operator $Q_{1}: \dot{V}_{5}\left(\Omega_{\delta}\right) \mapsto\left(\dot{V}_{5}\left(\Omega_{\delta}\right)\right)^{*}$, the form

$$
\left(\left(u_{\delta}, \psi\right) \in \dot{V}_{5}\left(\Omega_{\delta}\right) \times \dot{V}_{5}\left(\Omega_{\delta}\right)\right) \mapsto \int_{\Sigma_{\delta}^{s}} G_{\delta}^{1}\left(\left|u_{\delta}+\phi_{\delta}\right|^{3}\left(u_{\delta}+\phi_{\delta}\right)\right) \psi d s
$$

defines a weakly continuous operator $Q_{2}: \dot{V}_{5}\left(\Omega_{\delta}\right) \mapsto\left(\dot{V}_{5}\left(\Omega_{\delta}\right)\right)^{*}$ and the estimate (2.7) guarantees that the operator $Q_{1}+Q_{2}$ is coercive, then the boundary value problem (2.1) will have at least one weak solution $u_{\delta} \in \dot{V}_{5}\left(\Omega_{\delta}\right)$. Even more, the following estimate will hold:

$$
0 \leq\left(u_{\delta}+\phi_{\delta}\right) \leq M_{1} \text { a.e. on } \Omega_{\delta} .
$$

Let:

$$
\begin{aligned}
& h_{\delta}^{+}:=\left.\left(G_{\delta}^{2}\left(\left|T^{h}\right|^{3} T^{h}\right)+k_{3} T_{\delta}^{g}+P_{\delta}^{2}\left(\Sigma_{\delta}^{s}\right) F_{\delta} E_{\delta}^{2}\left(\Sigma_{\delta}^{s}\right)\left(\left|u_{\delta}+\phi_{\delta}\right|^{3}\left(u_{\delta}+\phi_{\delta}\right)\right)\right)\right|_{\Sigma_{\delta}^{+}}, \\
& h_{\delta}^{-}:=\left.\left(G_{\delta}^{2}\left(\left|T^{h}\right|^{3} T^{h}\right)+k_{3} T_{\delta}^{g}+P_{\delta}^{2}\left(\Sigma_{\delta}^{s}\right) F_{\delta} E_{\delta}^{2}\left(\Sigma_{\delta}^{s}\right)\left(\left|u_{\delta}+\phi_{\delta}\right|^{3}\left(u_{\delta}+\phi_{\delta}\right)\right)\right)\right|_{\Sigma_{\delta}^{-}}, \\
& h_{\delta}^{\prime}:=\left.\left(G_{\delta}^{2}\left(\left|T^{h}\right|^{3} T^{h}\right)+k_{3} T_{\delta}^{g}+P_{\delta}^{2}\left(\Sigma_{\delta}^{s}\right) F_{\delta} E_{\delta}^{2}\left(\Sigma_{\delta}^{s}\right)\left(\left|u_{\delta}+\phi_{\delta}\right|^{3}\left(u_{\delta}+\phi_{\delta}\right)\right)\right)\right|_{\Sigma_{\delta}^{\prime}}, \\
& h_{\delta}^{\prime \prime}:=\left.\left(G_{\delta}^{2}\left(\left|T^{h}\right|^{3} T^{h}\right)+k_{3} T_{\delta}^{g}+P_{\delta}^{2}\left(\Sigma_{\delta}^{s}\right) F_{\delta} E_{\delta}^{2}\left(\Sigma_{\delta}^{s}\right)\left(\left|u_{\delta}+\phi_{\delta}\right|^{3}\left(u_{\delta}+\phi_{\delta}\right)\right)\right)\right|_{\Sigma_{\delta}^{\prime \prime}},
\end{aligned}
$$




$$
\begin{aligned}
& \dot{h}_{\delta}^{+}:=\left.\left(G_{\delta}^{2}\left(\left|T^{h}\right|^{3} T^{h}\right)+k_{3} T_{\delta}^{g}+P_{\delta}^{2}\left(\Sigma_{\delta}^{s}\right) F_{\delta} E_{\delta}^{2}\left(\Sigma_{\delta}^{+}\right)\left(\left|u_{\delta}+\phi_{\delta}\right|^{3}\left(u_{\delta}+\phi_{\delta}\right)\right)\right)\right|_{\Sigma_{\delta}^{+}}, \\
& \dot{h}_{\delta}^{-}:=\left.\left(G_{\delta}^{2}\left(\left|T^{h}\right|^{3} T^{h}\right)+k_{3} T_{\delta}^{g}+P_{\delta}^{2}\left(\Sigma_{\delta}^{s}\right) F_{\delta} E_{\delta}^{2}\left(\Sigma_{\delta}^{-}\right)\left(\left|u_{\delta}+\phi_{\delta}\right|^{3}\left(u_{\delta}+\phi_{\delta}\right)\right)\right)\right|_{\Sigma_{\delta}^{-}}, \\
& h_{\delta}:=G_{\delta}^{2}\left(\left|T^{h}\right|^{3} T^{h}\right)+k_{3} T_{\delta}^{g}+P_{\delta}^{2}\left(\Sigma_{\delta}^{s}\right) F_{\delta} E_{\delta}^{2}\left(\Sigma_{\delta}^{s}\right)\left(\left|u_{\delta}+\phi_{\delta}\right|^{3}\left(u_{\delta}+\phi_{\delta}\right)\right), \\
& f_{\delta}^{+}:=\left.\left(G_{\delta}^{2}\left(\left|T^{h}\right|^{3} T^{h}\right)+k_{3} T_{\delta}^{g}\right)\right|_{\Sigma_{\delta}^{+}}, \quad f_{\delta}^{-}:=\left.\left(G_{\delta}^{2}\left(\left|T^{h}\right|^{3} T^{h}\right)+k_{3} T_{\delta}^{g}\right)\right|_{\Sigma_{\delta}^{-}} .
\end{aligned}
$$

Then (2.9) and properties of involved operators imply existence of a constant $M_{5}$ such that:

$$
\begin{aligned}
& h_{\delta}^{+} \in W_{\infty}^{1}\left(\Sigma_{\delta}^{+}\right), \quad h_{\delta}^{-} \in W_{\infty}^{1}\left(\Sigma_{\delta}^{-}\right), \quad h_{\delta}^{\prime} \in W_{\infty}^{1}\left(\Sigma_{\delta}^{\prime}\right), \quad h_{\delta}^{\prime \prime} \in W_{\infty}^{1}\left(\Sigma_{\delta}^{\prime \prime}\right), \\
& \dot{h}_{\delta}^{+} \in W_{\infty}^{1}\left(\Sigma_{\delta}^{+}\right), \quad \dot{h}_{\delta}^{-} \in W_{\infty}^{1}\left(\Sigma_{\delta}^{-}\right), \quad f_{\delta}^{+} \in W_{\infty}^{1}\left(\Sigma_{\delta}^{+}\right), \quad f_{\delta}^{-} \in W_{\infty}^{1}\left(\Sigma_{\delta}^{-}\right), \\
& h_{\delta}^{+} \geq 0, \quad \dot{h}_{\delta}^{+} \geq 0, \quad f_{\delta}^{+} \geq 0 \text { a.e. on } \Sigma_{\delta}^{+}, \\
& h_{\delta}^{-} \geq 0, \quad \dot{h}_{\delta}^{-} \geq 0, \quad f_{\delta}^{-} \geq 0 \text { a.e. on } \Sigma_{\delta}^{-}, \\
& h_{\delta}^{\prime} \geq 0 \text { a.e. on } \Sigma_{\delta}^{\prime}, \quad h_{\delta}^{\prime \prime} \geq 0 \text { a.e. on } \Sigma_{\delta}^{\prime \prime}, \\
& \left\|h_{\delta}^{+}\right\|_{W_{\infty}^{1}\left(\Sigma_{\delta}^{+}\right)} \leq M_{5}, \quad\left\|\dot{h}_{\delta}^{+}\right\|_{W_{\infty}^{1}\left(\Sigma_{\delta}^{+}\right)} \leq M_{5}, \quad\left\|h_{\delta}^{-}\right\|_{W_{\infty}^{1}\left(\Sigma_{\delta}^{-}\right)} \leq M_{5}, \\
& \left\|\dot{h}_{\delta}^{-}\right\|_{W_{\infty}^{1}\left(\Sigma_{\delta}^{-}\right)} \leq M_{5}, \quad\left\|h_{\delta}^{\prime}\right\|_{W_{\infty}^{1}\left(\Sigma_{\delta}^{\prime}\right)} \leq M_{5}, \quad\left\|h_{\delta}^{\prime \prime}\right\|_{W_{\infty}^{1}\left(\Sigma_{\delta}^{\prime \prime}\right)} \leq M_{5} .
\end{aligned}
$$

Moreover, the boundary value problem (2.1) can be rewritten in the following form:

$$
\begin{aligned}
& \int_{\Omega_{\delta}}\left(k_{1}\left(\nabla\left(u_{\delta}+\phi_{\delta}\right) \cdot \nabla \psi\right)+k_{2}\left(u_{\delta}+\phi_{\delta}\right)_{x_{1}} \psi\right) d v \\
&+\int_{\Sigma_{\delta}^{s}} \sigma \epsilon_{\delta}\left|u_{\delta}+\phi_{\delta}\right|^{3}\left(u_{\delta}+\phi_{\delta}\right) \psi d s+\int_{\Sigma_{\delta}^{s}} k_{3}\left(u_{\delta}+\phi_{\delta}\right) \psi d s \\
&=\int_{\Sigma_{\delta}^{s}} h_{\delta} \psi d s \quad \forall \psi \in \dot{V}_{5}\left(\Omega_{\delta}\right) .
\end{aligned}
$$

\section{Estimate for Gradients}

Let us choose a function $\eta_{\delta}: \Omega_{\delta} \mapsto \mathbb{R}$ defined as

$$
\eta_{\delta}(x):=\min \left\{\frac{\operatorname{dist}\left\{\Sigma_{\delta}^{i}, x\right\} l_{3}^{r}}{l_{1} \delta^{r}}, 1\right\}
$$

for some fixed $0<r<1$. Then it is easy to verify that

$$
\int_{\Omega_{\delta}}\left|\nabla \eta_{\delta}\right|^{2} d v \leq \frac{4 l_{3}^{r} l_{2}}{l_{1}} \delta^{1-r}
$$

If we define a function $\psi_{\delta}:=\left(u_{\delta}+\phi_{\delta}\right) \eta_{\delta}^{2}$, then $\psi_{\delta} \in \dot{V}_{5}\left(\Omega_{\delta}\right)$.

As functions $u_{\delta}+\phi_{\delta}, h_{\delta}, \psi_{\delta}$ are uniformly bounded with respect to $\delta$, then 


$$
\begin{aligned}
\left|\int_{\Sigma_{\delta}^{s}} \sigma \epsilon_{\delta}\right| u_{\delta}+\left.\phi_{\delta}\right|^{3}\left(u_{\delta}\right. & \left.+\phi_{\delta}\right) \psi_{\delta} d s \mid \\
& +\left|\int_{\Sigma_{\delta}^{s}} k_{3}\left(u_{\delta}+\phi_{\delta}\right) \psi_{\delta} d s\right|+\left|\int_{\Sigma_{\delta}^{s}} h_{\delta} \psi_{\delta} d s\right| \leq c_{4}
\end{aligned}
$$

For every chosen $\tau>0$ it follows that

$$
\begin{aligned}
& \mid \int_{\Omega_{\delta}} k_{2}\left(u_{\delta}+\phi_{\delta}\right)_{x_{1}} \psi_{\delta} d v|=| \int_{\Omega_{\delta}} k_{2}\left(u_{\delta}+\phi_{\delta}\right)_{x_{1}}\left(u_{\delta}+\phi_{\delta}\right) \eta_{\delta}^{2} d v \mid \\
& \leq k_{2} \tau \int_{\Omega_{\delta}}\left(u_{\delta}+\phi_{\delta}\right)_{x_{1}}^{2} \eta_{\delta}^{2} d v+\frac{k_{2}}{4 \tau} \int_{\Omega_{\delta}}\left(u_{\delta}+\phi_{\delta}\right)^{2} \eta_{\delta}^{2} d v
\end{aligned}
$$

and, therefore, if we put $\tau=\frac{k_{1}}{4 k_{2}}$, then, after taking into account uniform boundedness of $u_{\delta}+\phi_{\delta}$ with respect to $\delta$, we obtain

$$
\left|\int_{\Omega_{\delta}} k_{2}\left(u_{\delta}+\phi_{\delta}\right)_{x_{1}} \psi_{\delta} d v\right| \leq \frac{k_{1}}{4} \int_{\Omega_{\delta}}\left|\nabla\left(u_{\delta}+\phi_{\delta}\right)\right|^{2} \eta_{\delta}^{2} d v+c_{5}
$$

Again, for every chosen $\tau>0$ it follows that

$$
\begin{aligned}
\int_{\Omega_{\delta}} k_{1}\left(\nabla\left(u_{\delta}+\phi_{\delta}\right) \cdot \nabla \psi_{\delta}\right) d v \geq k_{1} \int_{\Omega_{\delta}}\left|\nabla\left(u_{\delta}+\phi_{\delta}\right)\right|^{2} \eta_{\delta}^{2} d v \\
\quad-k_{1} \tau \int_{\Omega_{\delta}}\left|\nabla\left(u_{\delta}+\phi_{\delta}\right)\right|^{2} \eta_{\delta}^{2} d v-\frac{k_{1}}{4 \tau} \int_{\Omega_{\delta}}\left|\nabla \eta_{\delta}\right|^{2}\left(u_{\delta}+\phi_{\delta}\right)^{2} d v
\end{aligned}
$$

and, if we put $\tau=1 / 4$, then after taking into account uniform boundedness of $u_{\delta}+\phi_{\delta}$ with respect to $\delta$ and (3.1), we will have

$$
\int_{\Omega_{\delta}} k_{1}\left(\nabla\left(u_{\delta}+\phi_{\delta}\right) \cdot \nabla \psi_{\delta}\right) d v \geq \frac{3 k_{1}}{4} \int_{\Omega_{\delta}}\left|\nabla\left(u_{\delta}+\phi_{\delta}\right)\right|^{2} \eta_{\delta}^{2} d v-c_{6} .
$$

Combining (2.10), (3.2) - (3.4), we finally get

$$
\int_{\Omega_{\delta}}\left|\nabla\left(u_{\delta}+\phi_{\delta}\right)\right|^{2} \eta_{\delta}^{2} d v \leq c_{7}
$$

\section{Main Estimate}

Let us choose functions $\dot{h}_{\delta}: \Omega_{\delta} \mapsto \mathbb{R}, \dot{\epsilon}_{\delta}: \Omega_{\delta} \mapsto \mathbb{R}$ defined as:

$$
\begin{aligned}
& \dot{h}_{\delta}\left(x_{1}, x_{2}, x_{3}\right):=\frac{\dot{h}_{\delta}^{+}\left(x_{1}, x_{2}\right)+\dot{h}_{\delta}^{-}\left(x_{1}, x_{2}\right)}{2}, \\
& \dot{\epsilon}_{\delta}\left(x_{1}, x_{2}, x_{3}\right):=\frac{\epsilon^{+}\left(x_{1}, x_{2}\right)+\epsilon^{-}\left(x_{1}, x_{2}\right)}{2} .
\end{aligned}
$$

Again, if we define a function 


$$
\psi_{\delta}:=\left(\sigma \dot{\epsilon}_{\delta}\left(u_{\delta}+\phi_{\delta}\right)^{4}+k_{3}\left(u_{\delta}+\phi_{\delta}\right)-\dot{h}_{\delta}\right) \eta_{\delta}^{2},
$$

then $\psi_{\delta} \in \dot{V}_{5}\left(\Omega_{\delta}\right)$.

As $\left\|\dot{\epsilon}_{\delta}\right\|_{W_{\infty}^{1}\left(\Omega_{\delta}\right)} \leq M_{3},\left\|\dot{h}_{\delta}\right\|_{W_{\infty}^{1}\left(\Omega_{\delta}\right)} \leq M_{5}$ and functions $u_{\delta}+\phi_{\delta}, h_{\delta}$ are uniformly bounded with respect to $\delta$, then, if we take into account (3.1), (3.5), then:

$$
\begin{gathered}
\int_{\Omega_{\delta}}\left|\nabla \psi_{\delta}\right|^{2} d v \leq \int_{\Omega_{\delta}} 48\left(\sigma \dot{\epsilon}_{\delta}\left(u_{\delta}+\phi_{\delta}\right)^{3}+k_{3}\right)^{2} \eta_{\delta}^{4}\left|\nabla\left(u_{\delta}+\phi_{\delta}\right)\right|^{2} d v \\
\quad+\int_{\Omega_{\delta}} 12 \sigma^{2}\left(u_{\delta}+\phi_{\delta}\right)^{8} \eta_{\delta}^{4}\left|\nabla \dot{\epsilon}_{\delta}\right|^{2} d v+\int_{\Omega_{\delta}} 12 \eta_{\delta}^{4}\left|\nabla \dot{h}_{\delta}\right|^{2} d v \\
+\int_{\Omega_{\delta}} 24\left(\sigma \dot{\epsilon}_{\delta}\left(u_{\delta}+\phi_{\delta}\right)^{4}+k_{3}\left(u_{\delta}+\phi_{\delta}\right)-\dot{h}_{\delta}\right)^{2} \eta_{\delta}^{2}\left|\nabla \eta_{\delta}\right|^{2} d v \leq c_{8}
\end{gathered}
$$

and

$$
\begin{gathered}
\int_{\Omega_{\delta}} k_{1}\left(\nabla\left(u_{\delta}+\phi_{\delta}\right) \cdot \nabla \psi_{\delta}\right) d v=k_{1} \int_{\Omega_{\delta}}\left(4 \sigma \dot{\epsilon}_{\delta}\left(u_{\delta}+\phi_{\delta}\right)^{3}+k_{3}\right) \eta_{\delta}^{2}\left|\nabla\left(u_{\delta}+\phi_{\delta}\right)\right|^{2} d v \\
+k_{1} \int_{\Omega_{\delta}}\left(\sigma\left(u_{\delta}+\phi_{\delta}\right)^{4} \eta_{\delta}^{2}\left(\nabla\left(u_{\delta}+\phi_{\delta}\right) \cdot \nabla \dot{\epsilon}_{\delta}\right)-\eta_{\delta}^{2}\left(\nabla\left(u_{\delta}+\phi_{\delta}\right) \cdot \nabla \dot{h}_{\delta}\right)\right) d v \\
+k_{1} \int_{\Omega_{\delta}} 2\left(\sigma \dot{\epsilon}_{\delta}\left(u_{\delta}+\phi_{\delta}\right)^{4}+k_{3}\left(u_{\delta}+\phi_{\delta}\right)-\dot{h}_{\delta}\right) \eta_{\delta}\left(\nabla\left(u_{\delta}+\phi_{\delta}\right) \cdot \nabla \eta_{\delta}\right) d v \\
\geq-k_{1}\left(\int_{\Omega_{\delta}} \sigma^{2}\left(u_{\delta}+\phi_{\delta}\right)^{8} \eta_{\delta}^{2}\left|\nabla \dot{\epsilon}_{\delta}\right|^{2} d v\right)^{1 / 2}\left(\int_{\Omega_{\delta}}\left|\nabla\left(u_{\delta}+\phi_{\delta}\right)\right|^{2} \eta_{\delta}^{2} d v\right)^{1 / 2} \\
-k_{1}\left(\int_{\Omega_{\delta}} \eta_{\delta}^{2}\left|\nabla \dot{h}_{\delta}\right|^{2} d v\right)^{1 / 2}\left(\int_{\Omega_{\delta}}\left|\nabla\left(u_{\delta}+\phi_{\delta}\right)\right|^{2} \eta_{\delta}^{2} d v\right)^{1 / 2} \\
-k_{1}\left(\int_{\Omega_{\delta}}\left(\sigma \dot{\epsilon}_{\delta}\left(u_{\delta}+\phi_{\delta}\right)^{4}+k_{3}\left(u_{\delta}+\phi_{\delta}\right)-\dot{h}_{\delta}\right)^{2}\left|\nabla \eta_{\delta}\right|^{2} d v\right)^{1 / 2} \\
\times\left(\int_{\Omega_{\delta}}\left|\nabla\left(u_{\delta}+\phi_{\delta}\right)\right|^{2} \eta_{\delta}^{2} d v\right)^{1 / 2} \geq-c_{9} \delta^{(1-r) / 2} . \quad \text { (4.2) }
\end{gathered}
$$

In addition

$$
\begin{aligned}
& \int_{\Omega_{\delta}} k_{2}\left(u_{\delta}+\phi_{\delta}\right)_{x_{1}} \psi_{\delta} d v \\
& \geq--k_{2} \int_{\Omega_{\delta}}\left|\left(u_{\delta}+\phi_{\delta}\right)_{x_{1}}\left(\sigma \dot{\epsilon}_{\delta}\left(u_{\delta}+\phi_{\delta}\right)^{4}+k_{3}\left(u_{\delta}+\phi_{\delta}\right)-\dot{h}_{\delta}\right)\right| \eta_{\delta}^{2} d v \\
& \geq-k_{2}\left(\int_{\Omega_{\delta}}\left(\sigma \dot{\epsilon}_{\delta}\left(u_{\delta}+\phi_{\delta}\right)^{4}+k_{3}\left(u_{\delta}+\phi_{\delta}\right)-\dot{h}_{\delta}\right)^{2} \eta_{\delta}^{2} d v\right)^{1 / 2} \\
&\left.\quad \times\left(\int_{\Omega_{\delta}}\left|\nabla\left(u_{\delta}+\phi_{\delta}\right)\right|^{2} \eta_{\delta}^{2} d v\right)\right)^{1 / 2} \geq-c_{10} \delta^{1 / 2}
\end{aligned}
$$

Now, let us estimate the integral

$$
-\int_{\Sigma_{\delta}^{s}} \sigma \epsilon_{\delta}\left|u_{\delta}+\phi_{\delta}\right|^{3}\left(u_{\delta}+\phi_{\delta}\right) \psi_{\delta} d s
$$


By using the Gauss formula

$$
\begin{aligned}
-\int_{\Sigma_{\delta}^{s}} \sigma \epsilon_{\delta} \mid u_{\delta} & +\left.\phi_{\delta}\right|^{3}\left(u_{\delta}+\phi_{\delta}\right) \psi_{\delta} d s \\
& =-\sigma \int_{\Omega_{\delta}}\left(\left(u_{\delta}+\phi_{\delta}\right)^{4} \psi_{\delta}\left(\frac{\dot{\epsilon_{\delta}}}{\delta} x_{3}+\frac{\epsilon^{+}-\epsilon^{-}}{2}\right)\right)_{x_{3}} d v \\
& -\int_{\Sigma_{\delta}^{\prime} \cup \Sigma_{\delta}^{\prime \prime}} \sigma \epsilon_{\delta}\left|u_{\delta}+\phi_{\delta}\right|^{3}\left(u_{\delta}+\phi_{\delta}\right) \psi_{\delta} d s
\end{aligned}
$$

and

$$
\begin{aligned}
& \int_{\Omega_{\delta}}\left(\left(u_{\delta}+\phi_{\delta}\right)^{4} \psi_{\delta}\left(\frac{\dot{\epsilon} \delta}{\delta} x_{3}+\frac{\epsilon^{+}-\epsilon^{-}}{2}\right)\right)_{x_{3}} d v \\
& \quad=\int_{\Omega_{\delta}} 4\left(u_{\delta}+\phi_{\delta}\right)^{3}\left(u_{\delta}+\phi_{\delta}\right)_{x_{3}} \psi_{\delta}\left(\frac{\dot{\epsilon} \delta}{\delta} x_{3}+\frac{\epsilon^{+}-\epsilon^{-}}{2}\right) d v \\
& \quad+\int_{\Omega_{\delta}}\left(u_{\delta}+\phi_{\delta}\right)^{4}\left(\psi_{\delta}\right)_{x_{3}}\left(\frac{\dot{\epsilon}_{\delta}}{\delta} x_{3}+\frac{\epsilon^{+}-\epsilon^{-}}{2}\right) d v+\frac{1}{\delta} \int_{\Omega_{\delta}} \dot{\epsilon}_{\delta}\left(u_{\delta}+\phi_{\delta}\right)^{4} \psi_{\delta} d v
\end{aligned}
$$

If we now take into account uniform boundedness of the functions $u_{\delta}+\phi_{\delta}, h_{\delta}$ with respect to $\delta$ and (3.5), then we get:

$$
\begin{gathered}
\int_{\Omega_{\delta}} 4\left(u_{\delta}+\phi_{\delta}\right)^{3}\left(u_{\delta}+\phi_{\delta}\right)_{x_{3}} \psi_{\delta}\left(\frac{\dot{\epsilon}_{\delta}}{\delta} x_{3}+\frac{\epsilon^{+}-\epsilon^{-}}{2}\right) d v \\
\geq-\left(\int_{\Omega_{\delta}} 16\left(\frac{\dot{\epsilon}_{\delta}}{\delta} x_{3}\right)^{2}\left(u_{\delta}+\phi_{\delta}\right)^{6}\left(\sigma \dot{\epsilon}_{\delta}\left(u_{\delta}+\phi_{\delta}\right)^{4}+k_{3}\left(u_{\delta}+\phi_{\delta}\right)-\dot{h}_{\delta}\right)^{2} \eta_{\delta}^{2} d v\right)^{1 / 2} \\
\times\left(\int_{\Omega_{\delta}}\left|\nabla\left(u_{\delta}+\phi_{\delta}\right)\right|^{2} \eta_{\delta}^{2} d v\right)^{1 / 2} \\
-\left(\int_{\Omega_{\delta}} 16\left(\frac{\epsilon^{+}-\epsilon^{-}}{2}\right)^{2}\left(u_{\delta}+\phi_{\delta}\right)^{6}\left(\sigma \dot{\epsilon}_{\delta}\left(u_{\delta}+\phi_{\delta}\right)^{4}+k_{3}\left(u_{\delta}+\phi_{\delta}\right)-\dot{h}_{\delta}\right)^{2} \eta_{\delta}^{2} d v\right)^{1 / 2} \\
\times\left(\int_{\Omega_{\delta}}\left|\nabla\left(u_{\delta}+\phi_{\delta}\right)\right|^{2} \eta_{\delta}^{2} d v\right)^{1 / 2} \geq-c_{11} \delta^{1 / 2}, \quad \text { (4.6) } \\
\int_{\Omega_{\delta}}\left(u_{\delta}+\phi_{\delta}\right)^{4}\left(\psi_{\delta}\right)_{x_{3}}\left(\frac{\dot{\epsilon}_{\delta}}{\delta} x_{3}+\frac{\epsilon^{+}-\epsilon^{-}}{2}\right) d v \\
\geq-\left(\int_{\Omega_{\delta}}\left(\frac{\dot{\epsilon}_{\delta}}{\delta} x_{3}\right)^{2}\left(u_{\delta}+\phi_{\delta}\right)^{8} d v\right)^{1 / 2}\left(\int_{\Omega_{\delta}}\left|\nabla \psi_{\delta}\right|^{2} d v\right)^{1 / 2} \\
-\left(\int_{\Omega_{\delta}}\left(\frac{\epsilon^{+}-\epsilon^{-}}{2}\right)^{2}\left(u_{\delta}+\phi_{\delta}\right)^{8} d v\right)^{1 / 2}\left(\int_{\Omega_{\delta}}\left|\nabla \psi_{\delta}\right|^{2} d v\right)^{1 / 2} \geq-c_{12} \delta^{1 / 2}
\end{gathered}
$$

and

$$
\int_{\Sigma_{\delta}^{\prime} \cup \Sigma_{\delta}^{\prime \prime}} \sigma \epsilon_{\delta}\left|u_{\delta}+\phi_{\delta}\right|^{3}\left(u_{\delta}+\phi_{\delta}\right) \psi_{\delta} \geq-c_{13} \delta .
$$


If we put (4.4) - (4.8) together, then

$$
-\int_{\Sigma_{\delta}^{s}} \sigma \epsilon_{\delta}\left|u_{\delta}+\phi_{\delta}\right|^{3}\left(u_{\delta}+\phi_{\delta}\right) \psi_{\delta} d s \leq-\frac{1}{\delta} \int_{\Omega_{\delta}} \sigma \dot{\epsilon}_{\delta}\left(u_{\delta}+\phi_{\delta}\right)^{4} \psi_{\delta} d v+c_{14} \delta^{1 / 2}
$$

Using similar techniques, we can also get the following estimates:

$$
\begin{aligned}
& \int_{\Sigma_{\delta}^{s}} h_{\delta} \psi_{\delta} d s \leq \frac{1}{\delta} \int_{\Omega_{\delta}} \dot{h}_{\delta} \psi_{\delta} d v+c_{15} \delta^{1 / 2} \\
& -\int_{\Sigma_{\delta}^{s}} k_{3}\left(u_{\delta}+\phi_{\delta}\right) \psi_{\delta} d s \leq-\frac{1}{\delta} \int_{\Omega_{\delta}} k_{3}\left(u_{\delta}+\phi_{\delta}\right) \psi_{\delta} d v+c_{16} \delta^{1 / 2} .
\end{aligned}
$$

Now, if we finally take into account (2.10), (4.2), (4.3), (4.9) - (4.11), then we get

$$
-c_{17} \delta^{(1-r) / 2} \leq \frac{1}{\delta} \int_{\Omega_{\delta}}\left(\dot{h}_{\delta}-\sigma \dot{\epsilon}_{\delta}\left(u_{\delta}+\phi_{\delta}\right)^{4}-k_{3}\left(u_{\delta}+\phi_{\delta}\right)\right) \psi_{\delta} d v
$$

or

$$
\int_{\Omega_{\delta}}\left(\sigma \dot{\epsilon}_{\delta}\left(u_{\delta}+\phi_{\delta}\right)^{4}+k_{3}\left(u_{\delta}+\phi_{\delta}\right)-\dot{h}_{\delta}\right)^{2} \eta_{\delta}^{2} d v \leq c_{17} \delta^{(3-r) / 2}
$$

If we choose a set $\Omega_{\delta}^{\prime}:=\left\{x \in \Omega_{\delta}: \eta_{\delta}(x)=1\right\}$, then (4.12) implies

$$
\int_{\Omega_{\delta}^{\prime}}\left(\sigma \dot{\epsilon}_{\delta}\left(u_{\delta}+\phi_{\delta}\right)^{4}+k_{3}\left(u_{\delta}+\phi_{\delta}\right)-\dot{h}_{\delta}\right)^{2} d v \leq c_{17} \delta^{(3-r) / 2}
$$

Since we have $\Omega_{\delta} \backslash \Omega_{\delta}^{\prime}=\left(0, \frac{l_{1} \delta^{r}}{l_{3}^{r}}\right) \times\left(-l_{2}, l_{2}\right) \times(-\delta, \delta)$, and functions $u_{\delta}+\phi_{\delta}, \dot{h}_{\delta}$ are uniformly bounded with respect to $\delta$, then

$$
\int_{\Omega_{\delta} \backslash \Omega_{\delta}^{\prime}}\left(\sigma \dot{\epsilon}_{\delta}\left(u_{\delta}+\phi_{\delta}\right)^{4}+k_{3}\left(u_{\delta}+\phi_{\delta}\right)-\dot{h}_{\delta}\right)^{2} d v \leq c_{18} \delta^{1+r}
$$

If we choose $r=\frac{1}{3}$, then estimates (4.13), (4.14) imply

$$
\int_{\Omega_{\delta}}\left(\sigma \dot{\epsilon}_{\delta}\left(u_{\delta}+\phi_{\delta}\right)^{4}+k_{3}\left(u_{\delta}+\phi_{\delta}\right)-\dot{h}_{\delta}\right)^{2} d v \leq c_{19} \delta^{4 / 3} \text {. }
$$

\section{Final Result}

Let us choose functions $\dot{\epsilon}: Q \mapsto \mathbb{R}, a: Q \times \mathbb{R} \mapsto \mathbb{R}, \dot{f}_{\delta}: Q \mapsto \mathbb{R}$ defined as:

$$
\begin{aligned}
& \dot{\epsilon}\left(x_{1}, x_{2}\right):=\frac{\epsilon^{+}\left(x_{1}, x_{2}\right)+\epsilon^{-}\left(x_{1}, x_{2}\right)}{2}, \quad a\left(x_{1}, x_{2}, \tau\right):=\sigma \dot{\epsilon}\left(x_{1}, x_{2}\right)|\tau|^{3} \tau+k_{3} \tau, \\
& \dot{f}_{\delta}\left(x_{1}, x_{2}\right):=\frac{f_{\delta}^{+}\left(x_{1}, x_{2}\right)+f_{\delta}^{-}\left(x_{1}, x_{2}\right)}{2}
\end{aligned}
$$


and an operator $\dot{F}_{\delta} \in \mathfrak{L}\left(L_{2}(Q), L_{2}(Q)\right)$ defined as

$$
\dot{F}_{\delta}:=\frac{P_{\delta}^{2}\left(\Sigma_{\delta}^{+}\right) F_{\delta} P_{\delta}^{1}\left(\Sigma_{\delta}^{+}\right)+P_{\delta}^{2}\left(\Sigma_{\delta}^{-}\right) F_{\delta} P_{\delta}^{1}\left(\Sigma_{\delta}^{-}\right)}{2} .
$$

As $a_{\tau}\left(x_{1}, x_{2}, \tau\right)>0$ for every $\left(x_{1}, x_{2}, \tau\right) \in Q \times \mathbb{R}$, then the theorem of implicit function guarantees, that there exists a function $b: Q \times \mathbb{R} \mapsto \mathbb{R}$, such that

$$
b\left(x_{1}, x_{2}, a\left(x_{1}, x_{2}, \tau\right)\right)=\tau,
$$

when $\left(x_{1}, x_{2}, \tau\right) \in Q \times \mathbb{R}$. But then we can define a new function $c: Q \times \mathbb{R} \mapsto \mathbb{R}$ by a mapping

$$
c\left(x_{1}, x_{2}, \tau\right):=\sigma \dot{\epsilon}\left(x_{1}, x_{2}\right)\left|b\left(x_{1}, x_{2}, \tau\right)\right|^{3} b\left(x_{1}, x_{2}, \tau\right) .
$$

As $b\left(x_{1}, x_{2}, 0\right)=0, c\left(x_{1}, x_{2}, 0\right)=0$ for every $\left(x_{1}, x_{2}\right) \in Q$ and

$$
\left|b_{\tau}\left(x_{1}, x_{2}, \tau\right)\right| \leq \frac{1}{k_{3}}, \quad\left|c_{\tau}\left(x_{1}, x_{2}, \tau\right)\right| \leq 1
$$

for every $\left(x_{1}, x_{2}, \tau\right) \in Q \times \mathbb{R}$, then, for measurable functions $u: Q \mapsto \mathbb{R}, v: Q \mapsto \mathbb{R}$ and almost every $\left(x_{1}, x_{2}\right) \in Q$ the following estimates will hold:

$$
\begin{aligned}
& \left|b\left(x_{1}, x_{2}, u\left(x_{1}, x_{2}\right)\right)-b\left(x_{1}, x_{2}, v\left(x_{1}, x_{2}\right)\right)\right| \leq \frac{\left|u\left(x_{1}, x_{2}\right)-v\left(x_{1}, x_{2}\right)\right|}{k_{3}}, \\
& \left|c\left(x_{1}, x_{2}, u\left(x_{1}, x_{2}\right)\right)-c\left(x_{1}, x_{2}, v\left(x_{1}, x_{2}\right)\right)\right| \leq\left|u\left(x_{1}, x_{2}\right)-v\left(x_{1}, x_{2}\right)\right|, \\
& \left|b\left(x_{1}, x_{2}, u\left(x_{1}, x_{2}\right)\right)\right| \leq \frac{\left|u\left(x_{1}, x_{2}\right)\right|}{k_{3}} \\
& \left|c\left(x_{1}, x_{2}, u\left(x_{1}, x_{2}\right)\right)\right| \leq\left|u\left(x_{1}, x_{2}\right)\right| .
\end{aligned}
$$

But then we get that the operators

$$
B(u):=b\left(x_{1}, x_{2}, u\left(x_{1}, x_{2}\right)\right), \quad C(u):=c\left(x_{1}, x_{2}, u\left(x_{1}, x_{2}\right)\right)
$$

map $L_{2}(Q)$ in $L_{2}(Q)$. Moreover, these operators are Lipschitz continuous.

Now let us consider the following equation

$$
v-\dot{F}_{\delta}(C(v))=f,
$$

where $v \in L_{2}(Q)$ is unknown variable and $f \in L_{2}(Q)$. Existence and unity of solutions for freely chosen right-hand side $f \in L_{2}(Q)$ of equation (5.1) easily can be proved by applying the theorem of contractive operators. Indeed, if we choose some functions $v_{1} \in L_{2}(Q), v_{2} \in L_{2}(Q)$, then

$$
\begin{aligned}
\left\|\dot{F}_{\delta}\left(C\left(v_{1}\right)\right)-\dot{F}_{\delta}\left(C\left(v_{2}\right)\right)\right\|_{L_{2}(Q)} & \leq\left\|\dot{F}_{\delta}\right\|_{\mathfrak{L}\left(L_{2}(Q), L_{2}(Q)\right)}\left\|C\left(v_{1}\right)-C\left(v_{2}\right)\right\|_{L_{2}(Q)} \\
& \leq c_{3}\left\|v_{1}-v_{2}\right\|_{L_{2}(Q)},
\end{aligned}
$$

and $0 \leq c_{3}<1$. 
As we know from the theorem of contractive operator, the sequence $\left\{v^{i} \in\right.$ $\left.L_{2}(Q)\right\}_{i \in \mathbb{N}}$ will converge to the solution $v^{*}$ of the equation (5.1) (for the fixed $f \in L_{2}(Q)$ ), if it is constructed in the following way:

$$
\begin{aligned}
v^{0} & :=f, \\
v^{i} & :=\dot{F}_{\delta}\left(C\left(v^{i-1}\right)\right)+f \quad 1 \leq i \leq \infty .
\end{aligned}
$$

Now, if we choose some $f_{1} \in L_{2}(Q), f_{2} \in L_{2}(Q)$, then we have:

$$
\begin{aligned}
&\left\|v_{1}^{1}-v_{2}^{1}\right\|_{L_{2}(Q)}=\left\|\dot{F}_{\delta}\left(C\left(f_{1}\right)\right)-\dot{F}_{\delta}\left(C\left(f_{2}\right)\right)+f_{1}-f_{2}\right\|_{L_{2}(Q)} \\
& \leq\left\|\dot{F}_{\delta}\right\|_{\mathfrak{L}\left(L_{2}(Q), L_{2}(Q)\right)}\left\|C\left(f_{1}\right)-C\left(f_{2}\right)\right\|_{L_{2}(Q)}+\left\|f_{1}-f_{2}\right\|_{L_{2}(Q)} \\
& \leq\left(c_{3}+1\right)\left\|f_{1}-f_{2}\right\|_{L_{2}(Q)}, \\
&\left\|v_{1}^{2}-v_{2}^{2}\right\|_{L_{2}(Q)}=\left\|\dot{F}_{\delta}\left(C\left(v_{1}^{1}\right)\right)-\dot{F}_{\delta}\left(C\left(v_{2}^{1}\right)\right)+f_{1}-f_{2}\right\|_{L_{2}(Q)} \\
& \leq\left\|\dot{F}_{\delta}\right\|_{\mathfrak{L}\left(L_{2}(Q), L_{2}(Q)\right)}\left\|C\left(v_{1}^{1}\right)-C\left(v_{2}^{1}\right)\right\|_{L_{2}(Q)}+\left\|f_{1}-f_{2}\right\|_{L_{2}(Q)} \\
& \leq c_{3}\left\|v_{1}^{1}-v_{2}^{1}\right\|_{L_{2}(Q)}+\left\|f_{1}-f_{2}\right\|_{L_{2}(Q)} \leq\left(c_{3}^{2}+c_{3}+1\right)\left\|f_{1}-f_{2}\right\|_{L_{2}(Q)},
\end{aligned}
$$

and therefore

$$
\left\|v_{1}^{*}-v_{2}^{*}\right\|_{L_{2}(Q)} \leq\left(\sum_{i=0}^{\infty}\left(c_{3}^{i}\right)\right)\left\|f_{1}-f_{2}\right\|_{L_{2}(Q)}=c_{20}\left\|f_{1}-f_{2}\right\|_{L_{2}(Q)} .
$$

As we found above, there exists an operator $\left(I-\dot{F}_{\delta}(C)\right)^{-1}: L_{2}(Q) \mapsto L_{2}(Q)$ and it is Lipschitz continuous. Therefore, we can define an operator $B\left(\left(I-\dot{F}_{\delta}(C)\right)^{-1}\right)$ : $L_{2}(Q) \mapsto L_{2}(Q)$, which is also Lipschitz continuous. Furthermore, it's Lipschitz constant is uniformly bounded with respect to $\delta$.

The operator $B\left(\left(I-\dot{F}_{\delta}(C)\right)^{-1}\right)$ can be used to transform (4.15) into the following estimate

$$
\int_{\Omega_{\delta}}\left(T_{\delta}-\tilde{T}_{\delta}\right)^{2} d v=\int_{\Omega_{\delta}}\left(\left(u_{\delta}+\phi_{\delta}\right)-\tilde{T}_{\delta}\right)^{2} d v \leq c_{21} \delta^{4 / 3}
$$

where the function $\tilde{T}_{\delta}: \Omega_{\delta} \mapsto \mathbb{R}$ is defined as

$$
\tilde{T}_{\delta}\left(x_{1}, x_{2}, x_{3}\right):=B\left(\left(I-\dot{F}_{\delta}(C)\right)^{-1}\right)\left(\dot{f}_{\delta}\right)\left(x_{1}, x_{2}\right) .
$$

As we see, $\tilde{T}_{\delta}$ can be used as a good approximation of temperature $T_{\delta}$ in $L_{2}\left(\Omega_{\delta}\right)$. We even know the rate of $\tilde{T}_{\delta}$ convergence to $T_{\delta}$ in $L_{2}\left(\Omega_{\delta}\right)$ norm.

Finally, it is important to note, that $\tilde{T}_{\delta}$ is a good temperature approximation even on the surface $\Sigma_{\delta}^{+} \cup \Sigma_{\delta}^{-}$(the proof is similar to previously used):

$$
\int_{\Sigma_{\delta}^{+} \cup \Sigma_{\delta}^{-}}\left(T_{\delta}-\tilde{T}_{\delta}\right)^{2} d s \leq c_{22} \delta^{1 / 3}
$$




\section{References}

[1] K. Birgelis. Optimal control in models with conductive-radiative heat transfer. Mathematical Modelling and Analysis, 8(1), 1 - 12, 2003.

[2] K. Birgelis. Boundedness of weak solutions of a conductive-radiative heat transfer problem. Acta Universitatis Latviensis, Ser. Mathematics, 2004. (accepted)

[3] A. Buikis and A. D. Fitt. A mathematical model for the heat treatment of glass fabric sheets. IMA Journal of Mathematics Applied in Business and Industry, 10, 55 - 86, 1999.

[4] N. Dunford and J. T. Schwartz. Linear operators. Interscience, New York, 1957.

[5] M. Laitinen and T. Tiihonen. Conductive-radiative heat transfer in grey materials. Report B6/2000, University of Jyvaskyla, 2000.

Temperatūros modeliavimas tarp plonų medžiagos lakštụ atsižvelgiant ị radiacijai laidžios šilumos pernešimą

K. Birgelis

Straipsnyje modeliuojamas temperat ${ }^{-}$uros pasiskirstymas tarp plonų medžiagos lakštu atsižvelgiant i radiacijai laidžios šlumos pernešimą. Nustatyta, kad temperat ${ }^{-u r a}$ tarp lakštu gali b־uti aproksimuojama $L_{2}$ normoje paprastos netiesinès operatorinès lygties sprendiniais. 